\title{
Généralités sur le cadre juridique du système de santé congolais : une analyse organisationnelle et fonctionnelle
}

\author{
Par Gloria LUBAKI SITA*
}

\begin{abstract}
This article reviews the Congolese health system as organized by the legislative and regulatory Act. There is no perfect model for a health system since each country organizes the system considering political, economic and social realities. The paper draws a parallel between, on the one hand, the political and administrative organisation of the Congolese state and the health structure, on the other hand, the adaptation of the health system into the social context of the country. It appears that territorial decentralization and the sharing of competences between the central government and the provinces could explain the pyramidal structure of the Heath system : The central government fixes the main orientations on health policy and the devolved services are aimed for implementing bodies, meanwhile the provinces only provide a technical assistance. According to the functional perspective, we describe a many-faceted system, due to the lack of a real social insurance coverage policies. Instead we find what we could call a system of adaptation made from private mutual insurance.
\end{abstract}

\section{Résumé}

Cet article s'intéresse au système de santé congolais tel que l'organisent les différents textes législatifs et réglementaires. Il n'existe pas de modèle parfait de système sanitaire puisque chaque pays l'organise eu égard aux considérations politique, économique et sociale qui l'identifient. Dans l'étude du cadre légal organisant le système congolais, nous faisons un parallélisme entre d'une part l'organisation politico-administrative de l'Etat congolais en général et la structure sanitaire ; et d'autre part, l'adaptation du système sanitaire aux réalités sociales du pays. Il apparaît que la décentralisation territoriale et les partages des compétences entre le pouvoir central et les provinces expliquent la structure pyramidale du système de santé congolais ; la santé étant un domaine de l'Etat, le pouvoir central donne les grandes orientations de la politique sanitaire et les services déconcentrés constituent des organes de mise en œuvre alors que les provinces n'assurent qu'une mission de relai. L'étude fonctionnelle fait voir un système multiforme, en attente de la mise en œuvre de la couverture sanitaire universelle.

* Doctorante à l'Université Technologique de Chemnitz (Allemagne) et coordonnatrice adjointe au ministère des affaires étranges de la République Démocratique du Congo (E-Mail : gloria.lubaki@s2018.tu-chemnitz.de). 


\section{Introduction}

Le système de santé congolais se caractérise d'une part par sa structure organisationnelle triangulaire, reflet du mode de gouvernance politique et administrative de l'Etat; et d'autre part, par sa structure fonctionnelle mettant l'accent sur la proximité et l'accès aux soins de santé primaire alors qu'il souffre encore d'un déficit en ce qui concerne l'assurance-maladie en particulier et la couverture sanitaire en générale. Le présent article se veut présenter le système de santé sous ces aspects organisationnel et fonctionnel. Il tend à répondre aux questions de savoir comment l'organisation politique a façonné la structure sanitaire et comment, en tant que Etat providence, les prestations sont organisées. En vrai, le système sanitaire se fait échos des réalités politiques, économiques et sociales de la République Démocratique du Congo. Les points forts sont un avantage et les défaillances reflètent ses faibles.

La santé telle que définie par l'OMS est un état de complet bien-être physique, mental et social et ne consiste pas seulement en une absence de maladie ou d'infirmité ${ }^{1}$. L'analyse de l'état de santé couvre donc plusieurs disciplines. Cet article n'a pas pour ambition d'épuiser le sujet. Il donne néanmoins un aperçu général sur le cadre réglementaire organisant le système de santé congolais. Le système de santé comprend toutes les organisations, institutions et ressources qui produisent des actions dont le but premier est d'améliorer la santé $^{2}$. Défini comme un ensemble des partenaires (service relié à un vaste réseau de personnes et d'interventions qui, d'une manière ou d'une autre, vont s'attacher à évaluer, entretenir et améliorer la santé) qui dispensent des services, les financent ou définissent des politiques pour les administrer ${ }^{3}$, le système de santé, ce réseau complexe, implique que soit délimitée les responsabilités des différents acteurs - publics et privés- chargés de mettre en œuvre les programmes du Gouvernement, de même que soit mis en évidence les attentes de la population.

Dans cet Etat décentralisé, la constitution a instauré deux régimes législatifs parallèles, entre le pouvoir central et les provinces, ayant des tunnels dans les matières à compétence concurrente. Les législations sur la santé, la protection sociale et les assurances relèvent de l'article 202 sur la compétence exclusive du pouvoir centrale ; et, la gestion du personnel médicale et des établissements au niveau provincial, l'organisation et la promotion des soins de santé primaires relèvent de l'article 204 attribuant à la compétence exclusive des provinces. L'article 203 se rapporte notamment à la prévention des épidémies et épizooties dangereuses pour la collectivité, matières à compétences concurrentes. Une telle organisation peut-elle contribuer à la performance du système sanitaire ? Nous répondons par l'af-

1 Müller Katharina, Kehler Jenni, Lechner Stefan, Neunsinger Sven, Rabe Florian, Transforming the Latvian health system : Accessibility of health services from a pro-poor perspective, German Development Institute, Bonn, 2005, p. 14.

2 OMS (2003), p. 105.

3 Art. 5 Loi n ${ }^{\circ}$ 18/035 du 13 décembre 2018 fixant les principes fondamentaux relatifs à l'organisation de la Santé publique, JO numéro spécial, 59ième année, 31 Décembre 2018. 
firmative. Puisque la politique générale du gouvernement insiste sur l'accès aux soins, une répartition d'attributions conduit à une implication et à la responsabilisation à tous les niveaux.

Dans un environnent international basé sur la confrontation entre le système fondé sur le marché et le système social, le système de santé congolais doit encore trouver son orientation. Le système sanitaire n'est pas que dépendant de la politique sociale du gouvernement, mais encore du modèle économique adopté. Un modèle économique basé sur le marché aura tendance à laisser la régulation aux forces du marché et l'Etat aura moins à intervenir. Cependant, la santé n'est pas n'importe quel marché. Les idéaux de protection sociales et d'actions humanitaires conduisent l'Etat à assurer un rôle de pilotage, comme c'est le cas dans un Etat providence. La faiblesse du système congolais réside dans la difficile protection de l'ensemble de la population contre les risques de maladie et la nécessité de soins de base. Il reste encore à définir le cadre réglementaire pour implémenter la couverture universelle. En fait, c'est une réforme de l'ensemble de la législation sociale qui s'impose.

\section{A. Influence du postulat politico- administrative sur l'organisation du système de santé congolais}

Il est de la responsabilité gouvernementale de mettre en place un système de santé performant $^{4}$; ce, afin d'assurer pleinement ses fonctions, c'est-à-dire garantir le bien-être de la population, prévenir des incapacités, maladies et mortalités précoces, promouvoir un meilleur état de la santé de la population ainsi qu'assurer la couverture, la qualité, l'efficience des services et la satisfaction des besoins de santé ${ }^{5}$. L'acte législatif ${ }^{6}$ qui a organisé le système de santé congolais jusqu'en 2018 souffrait de plusieurs lacunes, autant qu'il était devenu inadapté aux réalités politiques, économiques et sociales du pays. Avant le décret du 19 mars 1952, le système de santé congolais était modelé par différent textes règlementaires du

4 Le système est un terme à contour ambigu. Pour simplifier, un système renvoie l'idée d'un ensemble constitué de plusieurs éléments. Chaque Etat définit son système de santé au regard des réalités politiques, économiques et sociales. Il peut s'agir d'un ensemble d'organismes, institutions et ressources du secteur public et privé mandatés pour améliorer, maintenir ou rétablir la santé (charte de Tallinn), ou d'un ensemble coordonné de structures, de personnes et d'activités qui assurent la prévention des maladies, le maintien, la restauration et la promotion de la santé (Loi n¹8/035 du 13 Décembre 2018 fixant les principes fondamentaux relatifs à l'organisation de la santé publique). La performance d'un système de santé est fonction des indicateurs qui permettent d'en mesurer l'efficacité dans l'accomplissement de ses missions.

5 Art. 4 Loi 18/035 du 13 décembre 2018.

6 Le décret du 19 mars 1952 relatif à l'exercice de l'art de guérir n'a pas défini un cadre juridique organisant le système de santé congolais. La structure du texte se présente comme suit : Titre 1. Des médecins, titre II. Des dentistes, titre III. Des infirmiers, infirmières et assistantes infirmières coloniales, titre IV. Des agents sanitaires, titre V. Des accoucheuses, titre VI. Du personnel médical formé au Congo belge ou au Ruanda-Urundi, titre VII. De la pharmacie et de la droguerie, titre VIII. Généralités, titre IX. Des sanctions. 
Gouverneur Général ${ }^{7}$ tel qu'il peut être conclu à la lecture du décret du 19 juillet 1926 sur l'hygiène et salubrité publiques ${ }^{8}$.

Après l'indépendance, ni la loi fondamentale du 17 juin 1960 relative aux libertés publiques ni les différentes constitutions qui lui ont succédé jusqu'en 2006 n'ont eu la prétention de faire du droit à la santé un élément central. La législation sur l'art de guérir relevait de la compétence du pouvoir central dans la loi fondamentale de $1960^{9}$. De même, la constitution de 1964 avait réservé la législation sur la sécurité sociale, la législation médicale et l'art de guérir à la compétence exclusive du pouvoir centrale ${ }^{10}$. Ces différentes constitutions (excepté celle de 1967 qui avait instauré un Etat unitaire centralisé et les actes constitutionnels de Transitions) ont partagé les compétences législatives entre le pouvoir central et les provinces, selon que la matière tombe sous le domaine exclusif ou concurrent. La législation sur l'organisation et le fonctionnement du système de la santé était réservée à la compétence exclusive du pouvoir central, alors que la structure sanitaire et médicale au niveau provincial était organisée par la province.

7 Pour rappel, à l'époque du Congo-belge (1908-1960) l'inexistence d'une assemblée représentative du peuple faisait reposer l'essentiel de l'action législative entre les mains du Roi Belge statuant par voie de décret (art. 7) et le pouvoir exécutif exercé par voie de règlements et d'arrêtés (Art. 8). La loi du 18 octobre 1908 sur le gouvernement du Congo belge organisa l'administration de cette vaste colonie après son annexion par la Belgique.

8 Le gouverneur général est autorisé à établir par ordonnance des règlements pour empêcher l'introduction, prévenir l'éclosion et enrayer l'extension des maladies contagieuses, pour sauvegarder et améliorer l'hygiène publique, l'hygiène industrielle, l'hygiène infantile et pour assurer la police sanitaire maritime, fluviale, lacustre, terrestre et aérienne (art. $1^{\text {er }}$ décret du 19 juillet 1926 hygiène et salubrité publiques).

9 «Sans préjudice des dispositions particulières attribuant des compétences soit au pouvoir central soit au pouvoir provincial... les matières énumérées ci-après sont exclusivement attribuées au pouvoir central : 19. La législation sur l'art de guérir » (art. 219); " sans préjudice des dispositions particulières attribuant des compétences à la fois au pouvoir central et au pouvoir provincial, les matières énumérées ci-après relèvent expressément de l'un et de l'autre pouvoir : 1. La législation sociale et les règles relatives à la sécurité sociale ». (Art. 221).

10 Sans préjudice des autres dispositions de la présente Constitution, les matières suivantes sont de la compétence exclusive de la République : ...29 $9^{\circ}$ La législation du travail comprenant notamment les lois régissant les relations entre employeurs et travailleurs, la sécurité des travailleurs, les règles relatives à la sécurité sociale et, en particulier, le règles relatives aux assurances sociales et au chômage obligatoire; $32^{\circ}$ La législation médicale et l'art de guérir, la médecine préventive, notamment l'hygiène, la salubrité publique et la protection matérielle et infantile, la législation sur la profession de pharmacien, sur le commerce pharmaceutique, sur l'immigration et le transit, les règlements sanitaires bilatéraux et internationaux, la législation sur l'hygiène du travail, la répartition des médecins (art. 48 constitution du $1^{\text {er }}$ Août 1964). 17 ${ }^{\circ}$ L'affectation du personnel médical conformément au statut des agents de l'administration publique, l'établissement des programmes d'assainissement et de campagne de lutte contre les maladies endémo-épidémiques conformément au planning national, l'organisation des services d'hygiène et de prophylaxie provinciale, l'application et le contrôle de la législation médicale et pharmaceutique nationale, ainsi que l'organisation des services pharmaceutiques, la médecine curative, les entreprises médicales philanthropiques et missionnaires ainsi que les laboratoires médicaux. 
La Constitution du 18 février 2006, sans trop s'étendre sur la question de la protection et de la promotion de la santé, garantit le droit à la santé pour tous ${ }^{11}$. Aussi, à l'image du mode de gouvernance instauré par la constitution, le système de la santé publique congolais reflète le partage des compétences entre le niveau central et le niveau provincial selon le prescrit des articles 201 à 206 de la Constitution. Calqué sur cette structure, l'article 6 de la Loi ${ }^{\circ}$ 18/035 du 13 décembre 2018 instaure un système pyramidal à trois niveaux : le niveau central, le niveau intermédiaire ou provincial, le niveau périphérique ou opérationnel.

\section{La prise en charge de la stratégie générale de la santé publique par le pouvoir central}

La législation médicale et l'art de guérir, la médecine préventive, la législation sur la profession de pharmacien, sur le commerce pharmaceutique, sur l'immigration et le transit, les règlements sanitaires bilatéraux et internationaux, la législation sur l'hygiène du travail, la coordination technique des laboratoires médicaux et la répartition des médecins sont de la compétence exclusive du pouvoir centrale ${ }^{12}$. Il en est de même de la législation du travail qui inclut les lois régissant les relations entre employeurs et travailleurs, la sécurité des travailleurs, les règles relatives à la sécurité sociale ainsi que les règles relatives au chômage involontaire ${ }^{13}$. Figure aussi parmi les matières dévolues à la compétence exclusive du pouvoir central, la législation relative aux assurances ${ }^{14}$.

Le niveau central est chapeauté par trois structures : le ministre ayant la santé publique dans ses attributions comme autorité politique, le secrétariat général à la santé publique comme institution administrative centrale, et l'appui de l'Inspection générale à la santé. L'essentiel des fonctions revient au ministre de la santé qui doit conduire la politique du Gouvernement en matière sanitaire. Ce niveau définit les politiques, les stratégies, les normes et les directives ${ }^{15}$.

Le niveau central exerce une responsabilité normative et de régulation sur l'ensemble du système. L'autorité politique est chargée de la conception, élaboration et mise en œuvre de la politique sanitaire du Gouvernement. Conformément à l'ordonnance n 20/017 du 27 mars 2020 fixant les attributions des Ministères, les compétences du ministère de la Santé englobent, en sus de la formation médicale, l'organisation, la création et le contrôle des services publics médicaux et pharmaceutiques, l'hygiène et la santé publique, l'inspection et la prévention sanitaire et médicale, ainsi que les actions médicales humanitaires, la police sanitaire aux frontières (quarantaine humaine internationale), l'organisation, la réglementa-

11 «Le droit à la santé et à la sécurité alimentaire est garanti. La loi fixe les principes fondamentaux et les règles d'organisation de la santé publique et de la sécurité alimentaire »(art. 47 Const).

12 Art. 202.36h Const.

13 Art. 202.36E Const.

14 Art. 202.36A Const.

15 Art. 7 Loi 18/035 du 13 décembre 2018. 
tion et la promotion de la médecine traditionnelle, l'organisation du système de santé, l'élaboration des normes relatives à la santé.

Explicitement, la politique sanitaire et l'organisation du système sanitaire relèvent de la responsabilité du gouvernement central. Depuis 2001, les différents programmes de développement sanitaire élaborés par le gouvernement ont mis l'accent sur le développement et le renforcement de zones de santé ${ }^{16}$. En 2006, le Ministère de la Santé Publique s'est doté d'une stratégie de renforcement du système de santé, adoptée par le Gouvernement de la République comme stratégie sectorielle de réduction de la pauvreté. Elle constitue ainsi le fondement des nouvelles orientations politiques du pays en matière de santé ${ }^{17}$.

\section{Du contrôle et de la Supervision des infrastructures sanitaires par les provinces}

La décentralisation comme mode de gestion de la res publica conduit à un renforcement de la responsabilité des provinces dans l'accomplissement des missions régaliennes de l'Etat, matérialisée par le transfert des compétences du pouvoir central vers les institutions politiques et administratives des provinces. Dans le système des soins de santé, la constitution confère à la compétence exclusive des provinces, les matières relatives à la fonction publique provinciale et locale, l'organisation et le fonctionnement des services publics provinciaux,des établissements et entreprises publiques provinciaux dans le respect de la législation nationale ${ }^{18}$. Les compétences des provinces dans le fonctionnement du système sanitaire ne sont pas absolues. L'administration générale du système de santé reste sous la supervision du pouvoir central à qui il revient de définir les grandes stratégies et orientations de la politique sanitaire. L'action des pouvoirs provinciaux est limitée par le conformisme imposé de suivre les programmes nationaux. Ce conformisme est imposé tout d'abord par la constitution qui a délimité l'étendue des compétences des provinces. Ces limitations se trouvent dans l'étendue des compétences exclusives réservées aux provinces. Celles concernent l'élaboration des programmes d'assainissement et de campagne de lutte contre les maladies endémo- épidémiques conformément au plan national, l'organisation des services d'hygiène et de prophylaxie provinciale, l'application et le contrôle de la législation médicale et pharmaceutique nationale ${ }^{19}$.

Ce conformisme se situe aussi dans les restrictions des assemblées législatives provinciales à intervenir dans le fonctionnement du système sanitaire; tant le champ d'intervention des provinces revêt un caractère essentiellement administratif : l'organisation des services de la médecine curative, des services philanthropiques et missionnaires, des laboratoires médicaux et des services pharmaceutiques, l'organisation et la promotion des soins

16 Ecole de santé publique, Rapport sur l'Evaluation des prestations de services de soins de santé en RDC 2017-2018, Université de Kinshasa, Kinshasa, 2019, p. 2.

17 Ministère de la Santé, Plan nationale de développement sanitaire 2011-2015, Kinshasa, 2010, p. 30.

18 Art. 204 Const.

19 Art. 204.18 Const. 
de santé primaires. Par conséquent, ce niveau intermédiaire assure un rôle d'encadrement technique et la traduction des directives, stratégies, des politiques sous forme d'instructions et de fiches techniques pour faciliter la mise en œuvre au niveau des zones de santé ${ }^{20}$.

Le principe posé par la constitution est celui de la primauté de la législation nationale sur les actes provinciaux, mais si lesdits instruments juridiques provinciaux violent la constitution, le pouvoir central ne peut pas les annuler, car sa relation avec les provinces n'est pas hiérarchique ; et il n'a pas de pouvoir de contrôle sur leurs actions ${ }^{21}$. In casus specie, il existe en apparence de lien administratif hiérarchique entre l'administration centrale et les administrations provinciales, alors que les divisions provinciales ne sont pas des structures déconcentrées mais relèvent plutôt du service public provincial au sens de l'article 204.10 de la constitution. Les divisions provinciales de la santé sont des structures déconcentrées qui exercent les fonctions de contrôle et d'inspection au niveau provincial, sous la tutelle du gouverneur de province ${ }^{22}$.

L'apport des institutions administratives provinciales devrait être interprété dans le rôle de veiller au bon fonctionnement du système sanitaire, tant elles constituent le relai entre le régulateur du niveau central et les zones de santé. Dans les matières relevant de la compétence exclusive du pouvoir central, le Gouverneur de province coordonne et supervise les services qui relèvent de l'autorité du pouvoir central ${ }^{23}$. Par conséquent, les provinces sont chargées de la coordination, de l'appui, de l'encadrement, du contrôle, de la supervision, du suivi et de l'évaluation de l'ensemble des activités de santé24.

Toutefois in concreto, le niveau intermédiaire assume le rôle d'appui administratif que remplissant une fonction technique. Le cadre organique à ce niveau ne comprend pas en son sein des structures devant exercer la fonction relative à l'encadrement des zones de santé qui est une fonction essentielle pour ne pas dire la fonction la plus importante de ce niveau. Les structures du niveau intermédiaire passent leur temps à répondre aux exigences de celles du niveau central dont « elles sont issues » plutôt que de faire le travail d'encadrement des zones de santé 25 .

20 Ministère de la santé, Programme National de Développement Sanitaire 2016 - 2020 : vers la couverture sanitaire universel, Kinshasa, p. 22.

21 Balingene Kahombo, Regionalism under the constitution of the 18 February 2006: Progress and challenges, in ed. Charles M. Fombad and Nico Steyler, Decentralisation and constitutionalism in Africa, Oxford, 2019, p. 197.

22 Ministère de la Santé (2010), p. 23.

23 Dans l'hypothèse où il faudra considérer le terme Gouverneur comme englobant l'ensemble des actions du gouvernement provincial chapeautant l'administration provinciale. Ce raisonnement se peut justifier par une interprétation large de l'article 64 de la loi du 31 juillet 2008- Loi n08/012 portant principes fondamentaux relatifs à la libre administration des provinces. Par conséquent, l'article 65 pourra se lire « Dans l'exercice de sa mission de représentation du Gouvernement central et de coordination des services publics déconcentrés en province, le gouvernement provincial (Gouverneur de province) répond de ses actes devant le Gouvernement central.

24 Art. 8 Loi n ${ }^{\circ}$ 18/035 du 13 décembre 2018.

25 Ministère de la Santé (2010), p. 32. 


\section{Des services déconcentrés comme centre opérationnel}

La stratégie nationale sur le système de santé26 met l'accent sur le développent des zones de santé. Ces dernières sont des espaces géographiquement limités, couvrant une population théorique de 100.000 à 150.000 habitants avec un hôpital général de référence ${ }^{27}$. Les zones de santé ne sont pas rattachées aux entités territoriales décentralisées ; la création, l'organisation et le fonctionnement de la zone de santé sont de la compétence du ministre ayant la santé publique dans ses attributions. En effet, l'organisation du système sanitaire n'est pas tout à fait calquée sur la décentralisation territoriale de la RDC. Il faut analyser la zone de santé comme une structure déconcentrée de la province et non comme structure rattachée aux ETD; ce, pour prévenir toute demande de multiplication des zones de santé avec implantation d'hôpitaux de fortune ou de structures faisant fonction d'hôpital général de référence, d'une part et, d'autre part, pour régler la question relative à l'équité dans la distribution des ressources ${ }^{28}$. Toutefois, comme service public déconcentré, la zone de santé assure par délégation le relais sur le plan provincial et local des décisions prises par le pouvoir central, la province ou l'entité territoriale décentralisée ${ }^{29}$.

Le niveau opérationnel est chargé de la mise en œuvre de la stratégie des soins de santé primaires sous la supervision et l'encadrement de la province. Il se constitue du bureau central de la zone de santé, de l'hôpital général de référence, des centres de santé de référence, des centres de santé, des postes de santé, y compris d'autres établissements de santé publics et privés sous sa juridiction. Le centre opérationnel comprend différents établissements de santé tels que les postes de santé, les centres de santé, les centres de santé de références et les hôpitaux généraux ${ }^{30}$. Ces structures sont catégorisées en structures obligatoires d'une part - les centres de Santé et hôpitaux généraux de référence - et d'autre part, en structures facultatives que sont les centres de santé de référence et les postes de santé. L'on dénombre théoriquement 516 zones de santé chargées de la mise en œuvre de la stratégie des soins de

26 Ce document est présenté comme couvrant l'ensemble d'actions qui consistent à réorganiser le système de santé de manière à lui permettre à terme de couvrir l'ensemble de la population par des structures sanitaire qui offrent des soins de santé de base de qualité.

27 Ministère de la santé (2010), p. 23.

28 Idem, p. 33.

29 Art. 5.7 Loi n ${ }^{\circ}$ 16/001 du 03 mai 2016 fixant l'organisation et le fonctionnement des services publics du pouvoir central, des provinces et des entités territoriales décentralisées, J.O.N ${ }^{\circ} 11,2016$.

30 Le centre de santé dessert une population de 10000 habitants dans un rayon d'action de 5 à $8 \mathrm{kms}$ et comprend les services de réception, consultation, soins et d'observation, de maternité. L'hôpital général de référence offre les services de base suivants : Les services médicaux de base : Pédiatrie, Gynécologie obstétrique, médecine interne et la chirurgie. Un service de diagnostic : laboratoire et l'imagerie médicale (radiologie, échographie.), un service de réanimation et des soins intensifs, une pharmacie interne. En fonction de ses moyens et des compétences disponibles un hôpital peut en plus de services de base, développer tel ou tel autre spécialité médicale : Ophtalmologie, cardiologie, dermatologie, la chirurgie maxillo-faciale. 
santé primaires, avec 393 hôpitaux généraux de référence et 8.504 aires de santé planifiées dont 8.266 disposent d'un centre de santé ${ }^{31}$.

\section{B. Influence du modèle d'Etat providence sur la structure de prestation des soins}

Dans la logique de l'Etat providence, le politique du gouvernement doit assurer le bien-être de la population notamment en permettant aux individus et aux familles de faire face à des risques tels que la maladie, la vieillesse et le chômage ${ }^{32}$. L'Etat providence implique une responsabilisation du pouvoir public afin d'assurer la protection sociale des citoyens. Les risques mentionnés ci-haut correspondent aux 3 domaines politiques au cœur de l'État providence à savoir les soins de santé, les retraites et la politique du marché du travail ${ }^{33}$. La responsabilisation de l'Etat congolais afin d'assurer l'accès aux systèmes sanitaires trouve son fondement dans un droit reconnu et constitutionnellement garanti ; en effet, l'article 47 de la constitution dispose «Le droit à la santé et à la sécurité alimentaire est garanti». En outre, la loi nº18/035 du 13 décembre 2018 réaffirme ce droit en prévoyant l'instauration d'une couverture sanitaire universelle fondée sur les principes d'équité, d'assurance qualité des soins et de protection financière pour tous ${ }^{34}$.

Le système providentiel de l'Etat est ici analysé, d'une part, au travers la politique générale de protection sociale qui a gouverné les différents programmes et stratégies sanitaires, et dont l'accent fut mis sur la proximité des services et l'accès aux soins de santé primaire. Cette politique a comme avantage théorique (la volonté politique traduite sur les différents documents est souvent en inadéquation avec les réalités vécues par la population surtout dans les milieux ruraux) de permettre la prise en charge du grand nombre de la population en fournissant des soins basiques, c'est-à-dire ceux qui ne nécessitent pas de recours à des professionnels spécialisés. D'autre part, la couverture de la protection sociale sera étendue par la mise en place du système sanitaire universelle qui appellera certainement à certains aménagements du système actuel.

31 Ministère de la santé (2015), p. 23.

32 " A 'welfare state' is a state in which organized power is deliberately used (through politics and administration) in an effort to modify the play of market forces in at least three directions-first, by guaranteeing individuals and families a minimum income irrespective of the market value of their work or their property; second, by narrowing the extent of insecurity by enabling individuals and families to meet certain 'social contingencies' (for example, sickness, old age and unemployment) which lead otherwise to individual and family crises; and third, by ensuring that all citizens without distinction of status or class are offered the best standards available in relation to a certain agreed range of social services»(Briggs Asa, The welfare state in historical perspective, European journal of sociology, Vol. 2, 1961, p.288); cité aussi par Sell Christian, Die rechtliche Ausgestaltung des norwegischen Gesundheitssystems, Thèse, Friedrich-Schiller-Universität Jena, 2012, p. 6; MacGregor Susanne, Welfare: Theoretical and Analytical Paradigms, Working Paper 2014-13, United Nation Research Institute for social development, Geneva, September 2014, p.1.

33 MacGregor Susanne, Welfare: Theoretical and Analytical Paradigms, p. 2.

34 Art. 41 Loi n¹8/035 du 13 décembre 2018. 


\section{La politique de proximité et d'accès aux soins de santé primaire}

La proximité et l'accessibilité figurent parmi les critères gouvernant les prestations fournies aux usagers par tout service public, à côté de la participation et de la consultation ${ }^{35}$. La déconcentration des services médicaux doit contribuer à rendre les prestations les plus proches possibles des consommateurs. La subdivision du système congolais en zone de santé a le mérite de faciliter la couverture sanitaire. En effet, plus le nombre d'habitants par kilomètre couvert est réduit, moins la prise en charge pourra poser problème. Dans un pays où les voies de communication sont encore lacunaires, la déconcentration des services simplifie par conséquent la gestion des structures sanitaires et contribue à pallier à certains obstacles liés à l'éloignement des prestataires des soins.

Un autre palliatif est la communautarisation des prestations sanitaires. Les soins de santé primaire sont le premier niveau de contacts des individus, de la famille et de la communauté avec le système national de santé, rapprochant le plus possible les soins de santé des lieux où les gens vivent et travaillent ; ils constituent le premier élément d'un processus interrompu de protection sanitaire. Le système de santé congolais se veut inclusif : la population bénéficiaire des soins de santé participe, à titre bénévole, à toutes les activités relatives à la santé publique, et ce, à travers les organes de participation communautaire intervenant à différents niveaux de la pyramide sanitaire ${ }^{36}$. La participation de la communauté à l'action sanitaire peut prendre plusieurs formes, comme l'utilisation des services offerts par l'hôpital, la contribution et participation volontaire aux travaux d'entretien ou de construction de l'infrastructure. Des communautés organisées (confessions religieuses, les privés) peuvent être propriétaire d'un hôpital (hôpital privé) ou peuvent contractualiser avec l'Etat pour la gestion d'un hôpital général de référence dont les règles de jeu sont réglementées dans le document d'approche contractuelle ${ }^{37}$.

L'accessibilité à un système de santé signifie simplement que les patients ont la possibilité d'utiliser les services qui sont fournis par le système ${ }^{38}$. Alors que dans les pays à revenu élevé et moyen, les soins de santé primaires sont principalement considérés comme le premier niveau de soins; dans les pays à faible revenu, où l'accès aux soins de santé continue de poser des problèmes importants, ils sont davantage considérés comme une stratégie à l'échelle du système ${ }^{39}$. En se voulant accessible, le système congolais insiste sur la fourniture des soins de base devant en principe être à la bourse de la population.

La relation entre les soins aux patients et les fonctions de santé publique est l'une des caractéristiques de l'approche des soins de santé primaires ${ }^{40}$ (les prestations sanitaires se ca-

35 Art. 15 Loi n 16/001 du 03 mai 2016 fixant l'organisation et le fonctionnement des services publics du pouvoir central, des provinces et des entités territoriales décentralisées.

36 Art. 5 Loi n 18/035 du 13 décembre 2018.

37 Ministère de la Santé (2010), p. 34.

38 Katharina Müller; Jenni Kehler; Stefan Lechner; Sven Neunsinger; Florian Rabe, Op. Cit., p. 21.

39 Idem, p. 106.

40 OMC (2003), p. 106. 
tégorisent essentiellement en soins primaires, secondaires et tertiaires). Les soins primaires sont généralement dispensés par des médecins généralistes et comprennent des services de base, tels que le premier traitement, le diagnostic ou les services dentaires généraux. Les soins secondaires sont dispensés par des spécialistes ou des membres du personnel de l'hôpital. Dans la plupart des cas, les médecins généralistes orientent leurs clients vers des spécialistes pour un traitement avancé. Les soins tertiaires sont dispensés par des hôpitaux et des centres spécialisés ou par des médecins ayant une qualification unique ${ }^{41}$.

La déclaration de Alma-Ata de $1978^{42}$ a défini les soins de santé primaire comme des soins de santé essentiellement fondés sur des méthodes et des technologies pratiques, scientifiquement fondées et socialement acceptables, rendus universellement accessibles aux individus et aux familles de la communauté grâce à leur pleine participation et à un coût que la communauté et le pays peuvent se permettre de maintenir à chaque étape de leur développement dans un esprit d'autonomie et d'autodétermination.

La notion de soins de santé primaire doit être appréhendée dans une acception large qui inclut l'éducation concernant les problèmes de santé, la promotion des bonnes pratiques alimentaires, l'accès à l'eau potable et à un environnement sain, la protection maternelle et infantile, la prévention et le contrôle des endémies locales, traitement des maladies et lésions courantes et la fourniture de médicaments essentiels. Ce système basé sur les soins de santé primaires est structuré en 8 composantes : l'éducation concernant les problèmes de santé qui se posent ainsi que les méthodes de prévention et de lutte qui leur sont applicables; la promotion de bonnes conditions alimentaire et nutritionnelle; l'approvisionnement suffisant en eau saine et les mesures d'assainissement de base; la protection maternelle et infantile y compris la planification familiale; la vaccination contre les grandes maladies infectieuses; la prévention et le contrôle des endémies locales; le traitement des maladies et lésions courantes; la fourniture des médicaments essentiels ${ }^{43}$.

\section{Un système d'adaptation de couverture sanitaire ${ }^{44}$}

Le système de l'Etat providence implique pour ce dernier d'assurer pour la population le bénéfice d'une législation sociale favorable. La couverture universelle est instaurée par la

41 Katharina Müller; Jenni Kehler; Stefan Lechner; Sven Neunsinger; Florian Rabe, Op. Cit., p. 15.

42 La Conférence internationale sur les soins de santé primaires réunie à Alma-Ata en septembre 1978 s'est soldée par une déclaration qui affirme la santé comme un droit fondamental de l'être humain, que la promotion et la protection de la santé des peuples est la condition du progrès économique et social soutenu, appelle le gouvernement à élaborer des plans nationaux des politiques, des stratégies et des plans d'action visant à introduire et à maintenir les soins de santé primaires.

43 Ministère de la santé (2010) (2015).

44 La couverture des soins de santé désigne la proportion de la population qui reçoit un ensemble défini de produits et de services de santé dans le cadre de programmes publics et de l'assurance privée (OCDE, Panorama de la santé 2011 : Les indicateurs de l'OCDE, Paris, 2011, p. 123). 
section 2 de la loi de 2018 et donne à tout Congolais résidant sur le territoire national le droit à des prestations de soins de qualité à un coût accessible.

Le système universel s'oppose au système sélectif. Au sein même de système universel, il faudra voir si les programmes universels garantissent un minimum social ou s'ils s'efforcent de fournir un optimum social ${ }^{45}$. La différence entre les deux réside dans la force économique du Gouvernement. Dans un Etat économiquement fort, le gouvernement est apte à fournir conformément à sa politique sociale un optimum social, alors que les pays moins développés ne peuvent fournir, à la limite des ressources financières, qu'un minimum social. Dans le cadre de l'universalisme, l'ensemble de la population est bénéficiaire des prestations sociales en tant que droit fondamental ${ }^{46}$. L'article 42 dispose « le système de couverture sanitaire universelle garantit à tout Congolais résidant sur le territoire national le bénéfice de santé ».

La mise en œuvre d'un système universel implique un ensemble complet de services, incluant la sécurité sociale, l'éducation, les services sociaux personnels, les soins de santé et le logement ${ }^{47}$. Le décret de mise en œuvre de la couverture sanitaire universelle doit prendre le soin de définir les soins et services couverts.

Les soins doivent en principe être dispensés non pas en fonction de la richesse, mais en fonction des besoins. Aussi, se pose la question de la prise en charge du coût financier. En RDC, l'assurance-maladie n'est pas obligatoire. En fait, l'assurance-maladie n'est pas singularisée dans le code d'assurance, nous l'incluons au régime général des assurances de personnes ${ }^{48}$. La libéralisation tardive du secteur d'assurance tout comme la méfiance de la population à l'égard de la société nationale d'assurance doivent être prises en considération pour justifier le manque de couverture. Dans tout le cas, le régime d'assurance-maladie en RDC devrait se baser sur des primes payées par les souscriptions en contrepartie des services définis dans le contrat d'assurance. Une telle assurance suppose qu'il y a une convention au préalable entre l'assureur et le prestataire des soins de santé.

Dans le secteur public, la loi $n^{\circ} 16 / 013$ du 15 juillet 2016 portant statut des agents de carrière des services publics de l'Etat dispose en l'article 51 que l'agent encours de carrièrebénéficie comme avantages sociaux notamment les frais médicaux et soins de santé. Les frais médicaux et des soins couvre les soins de santé, chirurgicaux, obstétricaux, dentaires, ophtalmologiques et hospitaliers ainsi que lesmédicaments,leslunettes-médicales, des appareils d'orthopédie et de prothèse, des prothèses dentaires ${ }^{49}$. La couverture s'étend au conjoint et aux enfants à charge. Ce système, dans la pratique, souffre de certaines limitations, soit à cause du coût supporté par le trésor public (supporté jusqu'à certain seuil et

45 MacGregor Susanne, Op. Cit., p. 2.

46 Ibidem.

47 Ibidem.

48 Définie par la loi comme une assurance garantissant les risques dont la survenance dépend de la survie ou du décès ainsi que de l'incapacité et de l'invalidité de l'assuré.

49 Art. 55 Loi n ${ }^{\circ}$ 16/013 du 15 juillet 2016 portant statut des agents de carrière des services publics de l'Etat, JO 53 ${ }^{\text {ième }}$ année, numéro spécial du 03 Août 2016. 
l'agent prend en charge l'excédent), soit pour contourner la lenteur administrative dans le traitement du dossier, l'agent prend en charge la totalité des soins et réclame par la suite un remboursement jusqu'au seuil pris en charge par le trésor public. En outre, le trésor public ne couvre que les soins fournis auprès d'un établissement de soins caractère public et le seuil de couverture est parfois dérisoire.

Dans le secteur privé, le législateur impose à toute entreprise ou établissement d'assurer un service médical à ses travailleurs ${ }^{50}$. Ce service médical est soit fourni de manière autonome par l'établissement, soit l'employeur fait recours à un service commun à plusieurs entreprises, soit à une formation étrangère à l'entreprise ou à l'établissement ou à un médecin après avis du ministère du travail et de la prévoyance sociale ${ }^{51}$. Cette couverture médicale s'étend à la famille du travailleur et est entièrement supporté par l'employeur. Par conséquent, il ne peut déduire du salaire les frais occasionnés par les soins de santé. La prise en charge par l'employeur couvre les soins médicaux, dentaires, chirurgicaux, les frais pharmaceutiques et d'hospitalisation ainsi que les services ambulatoires et ophtalmologique ${ }^{52}$.

En dehors de la couverture offerte par le contrat de travail, et ceux qui peuvent se permettre de souscrire une assurance maladie privée, une grande partie de congolais ne bénéficie pas de protection sociale. Cela va des travailleurs indépendants et aux opérateurs œuvrant dans le secteur informel en passant par les travailleurs journaliers et ceux engagés sur base d'un contrat à durée déterminée, moins encore ceux qui sont en situation de chômage ainsi que les retraités ne bénéficiant pas du régime de pension.

La mutualisation de la santé peut être considérée comme le système qui vient couvrir le déficit de la couverture sanitaire. Les associations mutualistes ${ }^{53}$ peuvent être considérées comme des organismes privés ayant comme fondement d'assurer la protection sociale des adhérents et bénéficiaires et qui fonctionnent grâce aux cotisations des adhérents. Les mutuelles de santé sont régies par les principes fondamentaux gouvernant les associations mutualistes, tels qu'organisés par la loi n ${ }^{\circ} 17 / 002$ du 08 février $2017^{54}$. En soi, les mutuelles ont pour objectif de couvrir les bénéficiaires contre un risque qui affecterait leur situation sociale. La loi est à ce sujet large et laisse aux statuts de la mutuelle de déterminer les prestations couvertes. Aussi, les mutuelles peuvent avoir pour objet de couvrir les services de

50 Art. 177 Loi n $^{\circ}$ 015-2002 du 16 Octobre 2002 portant code du travail, JO, numéro spécial du 25 octobre 2002.

51 Art. 1 Arrêté départemental 01/76 du 21 janvier 1976 relatif au service médical ou sanitaire d'entreprise.

52 Art. 178 al. 1 Loi n ${ }^{\circ}$ 015-2002 du 16 Octobre 2002 portant code du travail.

53 La loi entend par mutuelle, le groupement des personnes physiques ou morales, de droit privé, à but non lucratif qui, au moyen des cotisations de ses membres, se propose de mener, dans l'intérêt de ceux-ci et/ou des personnes à leur charge, des actions de prévoyances, de solidarité et d'entraide (art. 4.4).

54 Elle constitue une loi spéciale par rapport à la loi nº 004/2001 du 20 juillet 2001 portant dispositions générales applicables aux associations sans but lucratif et aux établissements d'utilité publique, qui édicte des normes générales applicables aux organismes n'exerçant pas des opérations industrielles ou commerciales à titre principal. 
soins de santé en cas de maladie, d'accident, d'allocations familiales et de maternité, d'allocation de vieillesse et de décès ou d'autres prestations en faveur des membres adhérents et personnes à leur charge (bénéficiaires) selon que le détermineront les statuts ${ }^{55}$.

Les mutuelles organisent des services privés de prestations sociales en concurrence avec le système public de protection sociale, tout comme elles assument des prestations d'assurance, garantissant les bénéficiaires contre certains risques couverts par l'assurance des personnes. En général, les mutuelles couvrent les mêmes services relavant du régime de la sécurité sociale notamment la branche des risques professionnels, la branche des pensions, ou la branche des allocations familiales ${ }^{56}$. Les statuts fixent l'étendue des prestations prises en charge par la mutuelle, dépendamment du montant des cotisations des adhérents ; pour chaque branche couverte, la loi impose une gestion distincte ${ }^{57}$. Les mutuelles de santé en particulier sont conçues et développées comme une assurance-maladie nationale, offrant aux populations la possibilité d'accéder aux soins de santé de qualité à des prix décents ${ }^{58}$. L'adhésion à une mutuelle de santé donne accès aux soins de santé primaires préventifs et curatifs, soins hospitaliers et produits pharmaceutiques de même qu'aux soins spécialisés et dentaires $^{59}$, que l'adhérent pourra bénéficier à la fin d'une période probatoire fixée dans les statuts $^{60}$.

La mise en œuvre d'une couverture sanitaire universelle doit d'inscrire dans le cadre d'un système national d'assurance sociale complet, couvrant également les plans d'indemnisation des travailleurs, d'invalidité et de retraite et se fonder sur la solidarité ; de sorte que les riches payent un coût élevé pour supporter en partie la charge de la population en situation précaire. La participation citoyenne à la couverture universelle devra par exemple imposer une cotisation mensuelle proportionnelle au revenu. Ceci appellera certainement une mobilisation massive des ressources publiques et une augmentation de la part allouée à la santé publique dans le budget de l'Etat. La mise en œuvre d'une couverture santé universelle doit englober une spécificité croissante la couverture, la prestation de soins de santé et la qualité des soins. La couverture santé universelle fait de l'Etat socialement responsable d'assurer la santé pour tous. Avec comme conséquence, la mise en cause de l'Etat à travers ses organes en cas de non-respect de cette obligation ${ }^{61}$.

55 Art. 2 Loi n ${ }^{\circ} 17 / 002$ du 08 Février 2017 déterminant les principes fondamentaux relatifs à la mutuelle.

56 Nous avons fait le parallélisme entre l'art. 2 de la loi n ${ }^{\circ} 17 / 002$ du 08 Février 2017 déterminant les principes fondamentaux relatifs à la mutuelle et l'art. 1 du décret-loi du 29 Juin 1961 organisant la sécurité sociale.

57 Art. 3 Loi n $17 / 002$ du 08 Février 2017.

58 Art. 70 Loi n ${ }^{\circ} 7 / 002$ du 08 Février 2017.

59 Art. 74 Loi n $17 / 002$ du 08 Février 2017.

60 Art. 73 Loi n¹7/002 du 08 Février 2017.

61 L'État a donc un devoir formel de prendre soin de ses citoyens découlant en vertu de la constitution des articles 12 (protection par des lois) et 36 al.2 (protection sociale), en conjonction avec l'art. 11 (la dignité humaine), l'art. 16 et 47 (le droit à la vie et à la santé). Lire utilement Weide, 
La fonction des mutuelles de santé est un cas d'étude intéressant dans la planification d'une couverture sanitaire universelle. Elles permettent non seulement de regrouper les adhérents par centre d'intérêts et par région, mais en plus, elles prennent en charge les personnes évoluant tant dans le secteur formel qu'informel, public que privé ; de la sorte, les mutuelles de santé ont vocation à couvrir la majorité de la population. Dans la couverture par une mutuelle de santé, la loi impose une assurance-maladie obligatoire pour toute personne offrant la possibilité de rétention des cotisations à la source et une assurance maladie facultative pour les catégories des personnes dont on ne sait retenir des cotisations à la source $^{62}$.

\section{Conclusion : Pour une réforme du système de santé congolais}

Toute mesure tendant à la réforme du système sanitaire devra être une réponse à la question " comment renforcer le système sanitaire congolais? ». La réponse à cette question aura un impact multidimensionnel, car ne devant concerner non seulement la politique gouvernementale face à une crise sanitaire, comme tel est le cas, mais aussi impacter la politique sociale, environnementale et économique en générale. La crise actuelle due à la Covid-19 a montré sur plusieurs plans les déficits sanitaires, et ce même, dans les pays développés. Aussi, c'est l'occasion d'effectuer certaines réformes. En effet, les crises peuvent être décrites comme des moteurs introduisant des changements formels (par exemple, la création d'institutions) et informels (par exemple, la mobilisation de la société civile) ${ }^{63}$. Les pistes ici proposées ne sont pas exhaustives. Il s'agit pour notre part de clarifier le cadre légal et réglementaire régissant les établissements de santé (la gestion de l'institution que celles des ressources humaines), d'adopter une stratégie progressive pour une mise en place d'un système d'assurance-maladie universelle et dans une vision plus étendue, une révision de la législation sociale s'impose.

Une stratégie de réforme axée ces trois pistes a le mérite d'inclure les quatre types d'acteurs du système de santé. La première concerne les prestataires de soins, la deuxième ceux qu'on qualifie d'acheteurs et la troisième les consommateurs des services de soins. Dans tous les cas, les décideurs politiques sont nécessairement impliqués ${ }^{64}$.

Ursula, Law and the German universal healthcare system; a contemporary overview, German law journal: GLJ: open access to comparative, European, and international law; 6 (2005), 8, S. $1143-$ 1172; Online-Resource, p. 1146.

62 Art. 70 al. 2.

63 Bergner Susanne, the EU's Scope of Action in Global Health, Weinfelden: Stars - for Leaders of the Next Generation, 2020, p. 23.

64 „The relations between the different actors are characterised by the flow of services or payments.

Clients demand health care and use the health services that are being offered. The extent to which they use the latter depends not only on their need for health services, but also on their access to these services and their ability to pay for them. - Providers offer services, whether in a formal or informal context, and receive payment from clients or are being reimbursed by purchasers. - Purchasers contract services from providers and monitor their delivery to clients. Central or local go- 
En 2018, une étape primordiale a été franchie par l'actualisation du cadre législatif qui organise le système sanitaire congolais. La loi n 18/035 du 13 décembre 2018 fixant les principes fondamentaux relatifs à l'organisation de la santé publique a abrogé le texte vétuste et inadapté (décret du 19 mars 1952 relatif à l'art de guérir) qui ne correspondait plus à la réalité sociale congolaise. Elle établit un cadre d'organisation et du fonctionnement du système de santé publique clair et conforme à la structure administrative du pays, bien que plusieurs points doivent encore être approfondis ${ }^{65}$.

La loi donne quelques généralités sur les établissements des soins de santé. Il s'agit des structures servant un intérêt général de prévention et de prestation des soins. Ouvert aux prestataires publics et privés, la fourniture des soins se fait par conséquent sous forme concurrentielle.

La loi sur les établissements des soins de santé renforce la régulation de la fourniture des soins. Le marché de soins de santé est diversifié et comprend autant les institutions étatiques, privés et autres organismes religieuses ou associations sans but lucrative. Une telle diversité pourrait conduire à une concurrence dérégulée. La fourniture des soins de santé crée un marché confrontant les demandes des soins aux offres des soins. Contrairement à d'autres marchés, le marché des soins de santé présente des caractéristiques particulières ${ }^{66}$. Le marché des soins est basé sur la confiance du consommateur envers les prestataires. Ce qui appelle les autorités de contrôler la qualité des services fournis aux patients. En effet, le patient en tant que consommateur des services de santé devra recevoir non seulement toutes les informations nécessaires sur son état et son traitement, mais doit être assuré que les ser-

vernment, private companies, insurance funds or, in certain cases, clients can assume the role of purchasers. - Policymakers, or the so-called stewards, set the regulatory framework of the health system”. Müller, K., Kehler, J., Lechner, S., Neunsinger, S., et aliis, Op. Cit., p. 16.

65 Ce cadre légale est en construction du fait que la loi renvoie à plusieurs reprises à des textes d'application certaines matières. Il s'agit notamment du renvoi à un arrêté du ministre en ce qui concerne les modalités de collaboration entre les professionnels de santé et les organes de participation communautaire (art. 5); un arrêté devant fixer l'organisation et le fonctionnement des établissements des soins de santé (art. 12, repris mot à mot à l'art. 13); l'arrêté devant fixer les modalités d'application du droit à être soignée (art. 13); le décret du premier ministre devant déterminer les soins et services concernés par la couverture sanitaire universelle (art. 42); l'arrêté devant fixer la nomenclature du personnel de santé (art. 48); le décret du premier ministre devant fixer l'organisation et le fonctionnement du service national des plantes médicinales (art. 57); l'arrêté du ministre devant fixer les conditions d'exercice de la médecine traditionnelle (art. 60); l'arrêté devant déterminer les règles et procédures en matière de transfusion et transplantions (art. 68); le décret du premier ministre devant déterminer les conditions de la recherche biomédicale (art. 73) ainsi que celui déterminant le champ d'application du registre des sujets soumis à la recherche (art. 75); l'arrêté fixant les modalités de réalisation de l'assistance à la procréation (art. 80); l'arrêté fixant l'organisation et le fonctionnement du groupe technique consultatif national sur la vaccination (art. 88); art. 98; art. 105; art. 112; art. 115; art. 120; art. 128; Art. 129, pour ne citer que ceux-ci.

66 Lange Renate, Economic problems of health insurance: reforms and competition, Thèse, Universität Hohenheim, Hohenheim, 2016, p.2. 
vices fournis sont réellement efficaces et conformes ${ }^{67}$. La loi de 2018 contient des dispositions qui encadrent les relations entre patients et professionnels de santé68; et à travers le professionnel de santé, c'est la responsabilité de l'établissement des soins qui peut être engagée.

Le bénéfice d'une réglementation sur les établissements des soins assurera la protection des consommateurs. Une telle réglementation pourra résoudre les problèmes liés à la qualité, par exemple, à travers des règles de protection des clients telles que des normes de qualité minimales (contrôlées) ou des licences subordonnées à une formation régulière ${ }^{69}$. La relation médecin-patient est régie par le droit des contrats et les normes du droit de la responsabilité civile. Les prestataires assument une obligation de moyen, c'est-à-dire assurer les soins raisonnables; et en cas de négligence (fourniture d'un traitement défavorable), la tâche n'est pas aisé pour les consommateurs d'assumer la charge de la preuve. Les soins raisonnables sont une norme objective, reflétant les pratiques généralement acceptées et l'état des connaissances médicales au moment du traitement ${ }^{70}$. La loi devra donc définir l'étendue des responsabilités des prestataires.

La santé est une condition de base du développement humain, car l'état de santé d'un individu affecte directement sa capacité à gagner sa vie et à participer à la vie sociale ${ }^{71}$. Partant de ce constat, la réforme du système sanitaire doit s'étendre sur l'ensemble de la législation sur protection sociale. Cette réforme législative doit s'accompagner d'une mobilisation des ressources financières suffisantes pour prendre en charge une assurance sociale : d'une part, les prestations peuvent être directement fournies par le gouvernement à l'aide des recettes fiscales; d'autre part, mettre en place un régime des subventions financées par l'impôt. Le domaine de l'assure sociale est vaste, il englobe les programmes conçus principalement pour assurer contre les risques de santé, comme l'assurance maladie, l'assurance congé maladie, l'assurance accident, l'assurance soins de longue durée et l'assurance invalidité ; et les programmes qui assurent contre d'autres risques, comme l'assurance chômage, l'assurance retraite et l'assurance sociale spécifique à un pays ${ }^{72}$.

67 Conformément aux articles 17, "le malade a droit aux soins diligents et de qualité ", et 18, "Toute personne malade a droit aux soins qu'exige son état de santé dans le respect de sa dignité et, dans la mesure du possible, dans son cadre de vie habituel ».

68 Les articles 15 à 40 règlent le rapport entre le patient et le professionnel de santé.

69 Nuscheler Rober, On Competition and Regulation in Health Care Systems, Peter Lang International Academic Publishing Group, Frankfurt am Main, 2005, p. 5.

70 Weide Ursula, Law and the German universal healthcare system: a contemporary overview, 2005, p. 1159.

71 Müller, K., Kehler, J., Lechner, S., Neunsinger et aliis, op. Cit., p. 14:. "Health is a basic prerequisite for human development, since the individual health status directly affects one's ability to earn a living and to be involved in social participation".

72 Ziebarth, Nicolas R., Social insurance and health, IZA DiscussionPapers No. 10918, Bonn, p. 4. 


\section{Bibliographie}

1. Balingene Kahombo, Regionalism under the constitution of the 18 February 2006: Progress and challenges, in ed. Charles M. Fombad and Nico Steyler, Decentralisation and constitutionalism in Africa, Oxford University Press, Oxford, 2019, pp. 183211. https://www.researchgate.net/publication/344237220_Regionalism_under_the_C onstitution_of_18_February_2006_-Progress_and_challenges.

2. Bergner Susanne, The EU's Scope of Action in Global Health, Stars - for Leaders of the Next Generation, Weinfelden, May 2020, Online-Resource available on https:// www.the-stars.ch/wp-content/uploads/2019/04/Susan-BERGNER_The-EU\%E2\%80 $\% 99$ s-Scope-of-Action-in-Global-Health.pdf.

3. Briggs Asa, The welfare state in historical perspective, European journal of sociology, Vol. 2, 1961, ISSN: 0003-9756, 1474-0583, published online by Cambridge University Press: 28 July 2009.

4. Ecole de santé publique, Rapport sur l'Evaluation des prestations de services de soins de santé en RDC 2017-2018, Université de Kinshasa, Kinshasa, 2019.

5. Lange, Renate, Economic problems of health insurance: reforms and competition, Thèse, Universität Hohenheim, Hohenheim, 2016, http://opus.uni-hohenheim.de/vollt exte/2016/1293.

6. MacGregor Susanne, Welfare: Theoretical and Analytical Paradigms, Working Paper, United Nation Research Institute for social development, Geneva, 2014. https://w ww.unrisd.org/unrisd/website/document.nsf /(httpPublications)/ E5C803DD9F498AC8C1257D4E00547DAA?OpenDocument.

7. Ministère de la Santé, Plan nationale de développement sanitaire 2011-2015, Kinshasa, 2010.

8. Ministère de la santé, Programme National de Développement Sanitaire 2016 2020 : vers la couverture sanitaire universel, Kinshasa, 2015.

9. Müller Katharina, Kehler Jenni, Lechner Stefan, Neunsinger Sven, Rabe Florian, Transforming the Latvian health system: Accessibility of health services from a pro-poor perspective, German Development Institute, Bonn, 2005, ISBN 3-88985289-0. Online-resource http://edoc.vifapol.de/opus/volltexte/2012/3795/.

10. Nuscheler Rober, On Competition and Regulation in Health Care Systems, Thèse, Peter Lang International Academic Publishing Group, Frankfurt am Main, 2005, ISBN: 363175167, http://www.jstor.org/stable/j.ctv9hj6kj.

11. OCDE, Panorama de la santé 2011 : Les indicateurs de l'OCDE, Éd. OCDE, Paris, 2011, https://doi.org/10.1787/health_glance-2011-fr.

12. OMC, Rapport sur la santé dans le monde : Façonner l'avenir, Genève, 2003, ISBN 924256243 2, https://www.who.int/whr/2003/fr.

13. Sell Christian, Die rechtliche Ausgestaltung des norwegischen Gesundheitssystems, Thèse, Friedrich-Schiller-Universität Jena, 2012, Online: https://www.db-thueri ngen.de/receive/dbt_mods_00019737. 
14. Weide Ursula, Law and the German universal healthcare system: a contemporary overview, German law journal, 8, 2005, pp. 1143-1172, ISSN: 2071-8322, OnlineResource doi: 10.1017/S2071832200014206.

15. Ziebarth, Nicolas R., Social insurance and health, Discussion Papers, No. 10918, IZA, Institute of Labor Economics, Bonn, 2017, https://www.iza.org/publications/dp/ 10918. 